\title{
Qualitative analysis of solutions for a Kirchhoff-type parabolic equation with multiple nonlinearities
}

\author{
Erhan Pişkin*(D), Fatma Ekinci (D) \\ Dicle University, Department of Mathematics, 21280 Diyarbakır, Turkey
}

\begin{abstract}
In this work, the local and global existence of weak solutions by using the Faedo-Galerkin method, the finite time blow up of the weak solution with positive initial energy and the general decay of the solution are discussed. Finally, we consider the exponential growth of the solution with sufficient conditions. This work generalizes and improves earlier results in the literature, see [L.X. Truong and N. Van Y, On a class of nonlinear heat equations with viscoelastic term, Comput. Math. Appl., 2016] and [L.X. Truong and N. Van Y, Exponential growth with $L^{p}$-norm of solutions for nonlinear heat equations with viscoelastic term, Appl. Math. Comput., 2016].
\end{abstract}

Mathematics Subject Classification (2020). 35B44, 35G61, 35L75

Keywords. existence of solutions, blow up, exponential growth, decay, Kirchhoff-type equation

\section{Introduction}

In the present work, we investigate the following model of the Kirchhoff-type nonlinear heat equation with viscoelastic term and nonlinear source term

$$
\left(1+|u|^{q-2}\right) u_{t}-M\left(\|\nabla u\|^{2}\right) \Delta u+\int_{0}^{t} g(t-s) \Delta u(s) d s=f(u), \quad \text { in } \Omega \times[0, \infty),
$$

with boundary condition

$$
u(x, t)=0, \quad \text { on } \partial \Omega \times[0, \infty),
$$

and initial condition

$$
u(x, 0)=u_{0}(x), \quad \text { in } \Omega,
$$

where $\Omega \subset R^{n}(n \geq 1)$ is a bounded domain with smooth boundary $\partial \Omega, q$ is a positive constant, $M(s)=1+s^{\gamma},(\gamma>1$ and $s \geq 0)$. Also $g$ and $f(u)$ will be specified later.

In the case $M \equiv 1$, Truong and Van Y [11] considered the following problem

$$
\left(1+a|u|^{q-2}\right) u_{t}-\Delta u+\int_{0}^{t} g(t-s) \Delta u(s) d s=f(u) .
$$

\footnotetext{
*Corresponding Author.

Email addresses: episkin@dicle.edu.tr (E. Pişkin), ekincifatma2017@gmail.com (F. Ekinci)

Received: 09.12.2019; Accepted: 10.07.2020
} 
They showed the local and global existence of weak solutions by using the Faedo-Galerkin method. In addition, they proved the finite time blow-up and the decay of the weak solutions. Also, in [12], they investigated the result of exponentional growth of the weak solutions under the sufficient conditions of (1.4) with $f(u)=b|u|^{p-2} u$.

When $M \equiv 1$ and in the absence of $|u|^{q-2} u_{t}$ term, Eqs.(1.1) becomes the following problem

$$
u_{t}-\Delta u+\int_{0}^{t} g(t-s) \Delta u(s) d s=f(u) .
$$

In [9], Messaoudi investigated the blow up of the weak solution for the problem (1.5) with $f(u)=b|u|^{p-2} u$. Also, the equation (1.5) without memory term $(g=0)$ has been discussed by some authors (see $[1,2,5,7,10]$ ).

In detail, the present work is organized as follows: In next section, we present some notations, assumption and lemmas. In section 3, the existence are discussed by using Faedo-Galerkin's method that is similiar to used in [11]. In section 4, we prove blow-up result for solutions with positive energy. In section 5 , the exponential growth of solutions with positive initial energy is given. In last section, the decay property is studied.

\section{Preliminaries}

To state and prove our result, we need the following assumptions.

(A1) We suppose that $q$ belongs to the interval $\left(2, q^{*}\right)$, where

$$
q^{*}=\left\{\begin{array}{cc}
+\infty & \text { if } n=1,2, \\
\frac{2 n}{n-2} & \text { if } n \geq 3 .
\end{array}\right.
$$

(A2) The relaxation functions $g \in C^{1}$ is such that

$$
g(0) \geq 0, \quad g^{\prime}(s) \leq 0 \text { and } 1-\int_{0}^{\infty} g(s) d s=l>0 \text { for } s \geq 0 .
$$

(A3) The nonlinear source $f$ is a continuous function on $R$. Further, there is a constant $p$ such that

and

$$
2<p \leq q \quad \text { or } \quad q<p<2+\frac{2 q}{n}
$$

for some $C_{1}, C_{2}>0$ holds.

$$
|f(u)| \leq C_{1}+C_{2}|u|^{p-1}, \quad \forall u \in R .
$$

(A4) There is $p_{1} \in(\sqrt{2 p}, p)$ such that for all $m \in\left(0, p_{1}\right)$, there exists $\widehat{b}=\widehat{b}(m) \geq \frac{m b}{p}$ satisfying

$$
f(u) u-(p-m) F(u) \geq \widehat{b}|u|^{p}, \forall u \in R .
$$

(A5) $F(u) \leq \frac{b}{p}|u|^{p}, \forall u \in R$, where $b$ is a positive constant and

$$
2<p \leq q \text { or } q<p<2+\frac{2 q}{n} .
$$

(A6) $\int_{0}^{\infty} g(s) d s<\frac{\widehat{p}-2}{\widehat{p}-2+\widehat{p}^{-1}}$ where $\widehat{p}=\frac{p_{1}^{2}}{p}$.

Next, we introduce the energy function as follows

$$
E(t)=\frac{1}{2}\left[(g \diamond \nabla u)(t)+\frac{1}{\gamma+1}\left(\|\nabla u\|^{2(\gamma+1)}\right)\right]+\frac{1}{2}\left(1-\int_{0}^{t} g(s) d s\right)\|\nabla u(t)\|^{2}-\int_{\Omega} F(u) d x
$$

where $(g \diamond w)(t)=\int_{0}^{t} g(t-s)\|w(s)-w(t)\|_{2}^{2} d s$ and $F(u)=\int_{0}^{u} f(\xi) d \xi$.

We shall give some notations and lemmas throughout this work.

$$
\|\cdot\|_{2}=\|\cdot\|_{L^{2}} \quad \text { and } \quad\|\cdot\|_{p}=\|\cdot\|_{L^{p}} .
$$


Lemma 2.1 ([6]). Suppose that $2 \leq q<p<q^{*}$ and

$$
\theta=\left(\frac{1}{q}-\frac{1}{p}\right)\left(\frac{1}{n}-\frac{1}{2}+\frac{1}{q}\right)^{-1}
$$

hold. Then $\|w\|_{p} \leq C\|\nabla w\|_{2}^{\theta}\|w\|_{q}^{1-\theta}$ for all $w \in H_{0}^{1}(\Omega)$.

Lemma 2.2. Let $(A 2)$ and (2.1) hold. Then, $E(t)$ is a nonincreasing function for on $[0, \infty)$ and

$$
E^{\prime}(t) \leq 0
$$

Proof. Multiplying Eq.(1.1) by $u_{t}$, and integrating them over $\Omega$, then summing, we have

$$
E^{\prime}(t)=-\left(\frac{1}{2} g(t)\|\nabla u\|^{2}-\frac{1}{2}\left(g^{\prime} \diamond \nabla u\right)(t)+\int_{\Omega} u_{t}^{2} d x+\int_{\Omega}|u|^{q-2} u_{t}^{2} d x\right) \leq 0 .
$$

\section{Local existence and global existence}

Firstly, we introduce the definition of weak solutions for the problem (1.1).

Definition 3.1. The function $u(x, t)$ is a weak solution of problem $(1.1)$ on $\Omega \times[0, T)$ if $u \in L^{\infty}\left(0, T ; W_{0}^{1,2(\gamma+1)}(\Omega)\right), \quad u_{t} \in L^{2}\left(0, T ; L^{2}(\Omega)\right), \quad\left(|u|^{q / 2}\right)_{t} \in L^{2}(\Omega \times(0, T))$, satisfying $u(x, 0)=u_{0}(x)$ and

$$
\begin{aligned}
& \int_{\Omega}\left(u_{t}+|u|^{q-2} u_{t}\right) v d x+\int_{\Omega} M\left(\|\nabla u\|^{2}\right) \nabla u(t) \nabla v d x-\int_{0}^{t} g(t-s) \int_{\Omega} \nabla u(s) \nabla v d x d s \\
= & \int_{\Omega} f(u) v d x,
\end{aligned}
$$

for all $v \in L^{2}\left(0, T ; W_{0}^{1,2(\gamma+1)}(\Omega)\right)$ and for a.e. $t \in[0, T]$.

Lemma $3.2([11])$. Let $2<q<p<q^{*}$. For each $\varepsilon>0$, there exists a positive constant $C_{\varepsilon}$ such that

$$
\|w\|_{p}^{p} \leq \varepsilon\|\nabla w\|_{2}^{2}+C_{\varepsilon}\|w\|_{q}^{\alpha q}
$$

for all $w \in H_{0}^{1}(\Omega) \cap L^{q}(\Omega)$, where

$$
\alpha=\frac{2 p(1-\theta)}{q(2-p \theta)}, \quad \theta=\left(\frac{1}{q}-\frac{1}{p}\right)\left(\frac{1}{n}-\frac{1}{2}+\frac{1}{q}\right)^{-1} .
$$

Now, we shall find global existence and local existence in the following theorem that.

Theorem 3.3. Let (A1), (A2) and (A3) hold. If

$$
2<p \leq q,
$$

then for each $u_{0} \in W_{0}^{1,2(\gamma+1)}(\Omega)$, the problem (1.1) has a global weak solution of class

$$
u \in L^{\infty}\left(0, T ; W_{0}^{1,2(\gamma+1)}(\Omega)\right), \quad u_{t} \in L^{2}\left(0, T ; L^{2}(\Omega)\right), \quad\left(|u|^{q / 2}\right)_{t} \in L^{2}(\Omega \times[0, T)),
$$

for all $T>0$. If

$$
q<p<2+\frac{2 q}{n},
$$

then, for any $u_{0} \in W_{0}^{1,2(\gamma+1)}(\Omega)$, there is $T_{*}>0$ such that the problem (1.1) has a weak solution of class

$$
u \in L^{\infty}\left(0, T ; W_{0}^{1,2(\gamma+1)}(\Omega)\right), \quad u_{t} \in L^{2}\left(0, T ; L^{2}(\Omega)\right), \quad\left(|u|^{q / 2}\right)_{t} \in L^{2}(\Omega \times[0, T)),
$$

for all $T \in\left(0, T_{*}\right)$. 


\section{Proof. Approximate solutions}

We establish the existence of weak solution of the problem (1.1) via the Faedo-Galerkin's method. Let $\left\{v_{j}\right\}_{j=1}^{\infty}$ forms be an orthonormal basis for $L^{2}(\Omega)$ as well as $W_{0}^{1,2(\gamma+1)}(\Omega)$. Let $W_{k}=\operatorname{span}\left\{v_{1}, v_{2}, \ldots, v_{k}\right\}$ and $u_{0 k}$ be an element of $W_{k}$ given by

$$
u_{0 k}=\sum_{j=1}^{k} \alpha_{k j} v_{j} \longrightarrow u_{0} \text { strongly in } W_{0}^{1,2(\gamma+1)}(\Omega), \text { as } k \mapsto \infty .
$$

We search for an approximate solution of problem (1.1) such that

$$
u_{k}(t)=\sum_{j=1}^{k} c_{k j}(t) v_{j}
$$

where $c_{k j}(1 \leq j \leq k)$ satisfy the system of integro-differential equations

$$
\begin{aligned}
& \int_{\Omega}\left(1+\left|u_{k}(t)\right|^{q-2}\right) u_{k}^{\prime}(t) v_{i} d x+\int_{\Omega} M\left(\left\|\nabla u_{k}(t)\right\|^{2}\right) \nabla u_{k}(t) \nabla v_{i} d x \\
& -\int_{\Omega} \int_{0}^{t} g(t-s) \nabla u_{k}(s) \nabla v_{i} d s d x \\
= & \int_{\Omega} f\left(u_{k}(t)\right) v_{i} d x, \quad i=1,2, \ldots, k
\end{aligned}
$$

where the initial conditions

$$
c_{k j}(0)=\alpha_{k j}, \quad j \in\{1,2, \ldots, k\} .
$$

\section{A priori estimate I}

Multiplying (3.3) by $c_{k i}(t)$ and take the sum respect to $i$ from 1 to $k$ and then integrating on $[0, t]$. Afterwards we get

$$
A_{k}(t)=A_{k}(0)+\int_{0}^{t} d s \int_{0}^{s} g(s-\tau) \int_{\Omega} \nabla u_{k}(\tau) \nabla u_{k}(s) d x d \tau+\int_{0}^{t} d s \int_{\Omega} f\left(u_{k}(s)\right) u_{k}(s) d x,
$$

where

$$
A_{k}(t)=\frac{1}{2}\left\|u_{k}(t)\right\|^{2}+\frac{1}{q}\left\|u_{k}(t)\right\|_{q}^{q}+\int_{0}^{t}\left(\|\nabla u(s)\|^{2(\gamma+1)}+\|\nabla u(s)\|^{2}\right) d s .
$$

As applying the embedding $W^{1,2(\gamma+1)}(\Omega) \hookrightarrow L^{p}(\Omega), p \geq 1$, it follows from (3.1) that

$$
A_{k}(0)=\frac{1}{2}\left\|u_{0 k}\right\|^{2}+\frac{1}{q}\left\|u_{0 k}\right\|_{q}^{q} \leq C_{3}
$$

for positive constant $C_{3}$ not dependent on $k$. To estimate the second term on right-hand side of (3.5), we use Hölder and Young inequality,

$$
\begin{aligned}
& \int_{0}^{t} d s \int_{0}^{s} g(s-\tau) \int_{\Omega} \nabla u_{k}(\tau) \nabla u_{k}(s) d x d \tau \\
\leq & \frac{1}{2} \int_{0}^{t}\left\|\nabla u_{k}(s)\right\|^{2} d s+\frac{1}{2} \int_{0}^{t}\left(\int_{0}^{s} g(s-\tau)\left\|\nabla u_{k}(\tau)\right\|_{2} d \tau\right)^{2} d s \\
\leq & \frac{1}{2}(2-l) \int_{0}^{t}\left\|\nabla u_{k}(s)\right\|_{2}^{2} d s .
\end{aligned}
$$

Firstly, we take into account the case

$$
2<p \leq q .
$$


Then, by $(A 3)$ and using the embedding $L^{q}(\Omega) \hookrightarrow L^{p}(\Omega), p \leq q$, we obtain

$$
\begin{aligned}
\int_{0}^{t} d s \int_{\Omega} f\left(u_{k}(s)\right) u_{k}(s) d x & \leq \int_{0}^{t} \int_{\Omega}\left(C_{1}+C_{2}\left|u_{k}(s)\right|^{p-1}\right)\left|u_{k}(s)\right| d x d s \\
& \leq C_{4} \int_{0}^{t} A_{k}(s) d s+C_{5},
\end{aligned}
$$

where $C_{4}, C_{5}>0$, not dependent of $k$.

Combining (3.5)-(3.6), (3.7)-(3.8) and using the Gronwall-Bellman-Bihari type inequality (see [3], Theorem 21), we obtain

$$
A_{k}(t) \leq C_{T}, \quad \text { for any } t \in[0, T], \text { for all } k \in N,
$$

for all $T>0$.

Now, we consider the case

$$
q<p<2+\frac{2 q}{n}
$$

In the same way, from the assumption $(A 3)$ and Lemma 3.2, we get

$$
\int_{0}^{t} d s \int_{\Omega} f\left(u_{k}(s)\right) u_{k}(s) d x \leq \varepsilon \int_{0}^{t}\left\|\nabla u_{k}(s)\right\|^{2} d s+C_{6} \int_{0}^{t} A_{k}^{\alpha}(s) d s+C_{7},
$$

where $\alpha=\frac{2 p(1-\theta)}{q(2-p \theta)}>1$, and $C_{i}(i=6,7)$ are positive constants that independent on $k$. Thus, if we pick $\varepsilon$ to be sufficiently small, it follows from (3.5), (3.6), (3.7), (3.9) that

$$
A_{k}(t) \leq C_{8}+C_{9} \int_{0}^{t} A_{k}^{\alpha}(s) d s
$$

where $C_{8}$ and $C_{9}$ are positive constants that independent on $k$. Therefore, applying the Gronwall-Bellman-Bihari type inequality, there exists a positive constant $T_{*}>0$ such that, for all $T \in\left(0, T_{*}\right)$,

$$
A_{k}(t) \leq C_{T_{*}}, \forall t \in[0, T], \forall k \in N .
$$

So, in all the cases considered above, we obtained estimates of the form

$$
A_{k}(t) \leq \text { constant independent of } k, \forall t \in[0, T] ;
$$

furthermore, if $2<p \leq q$ then the quantity $T>0$ is arbitrarily large and fixed, whereas if

$$
q<p<2+\frac{2 q}{n}
$$

the quantity $T>0$ is rather small $\left(T \in\left(0, T_{*}\right)\right.$, for some $\left.T_{*}>0\right)$.

\section{A priori estimate II}

Now, multiplying the equation of (3.3) by $c_{k i}^{\prime}(t)$, summing with respect to $i$ and integrating over $(0, t)$, we get

$$
\begin{aligned}
& \int_{0}^{t}\left\|u_{k s}\right\|^{2} d s+\int_{0}^{t} \int_{\Omega}\left|u_{k}\right|^{q-2} u_{k s}^{2} d x d s+\psi_{k}(t) \\
= & \psi_{k}(0)-\frac{1}{2} \int_{0}^{t} g(s)\left\|\nabla u_{k}(s)\right\|^{2} d s \\
& +\frac{1}{2} \int_{0}^{t}\left(g^{\prime} \diamond \nabla u_{k}\right)(s) d s-\int_{\Omega} F\left(u_{0 k}\right) d x+\int_{\Omega} F\left(u_{k}\right) d x,
\end{aligned}
$$

where

$$
\begin{aligned}
\psi_{k}(t)= & \frac{1}{2}\left(1-\int_{0}^{t} g(s) d s\right)\left\|\nabla u_{k}(t)\right\|^{2}+\frac{1}{2}\left(g \diamond \nabla u_{k}\right)(t) \\
& +\frac{1}{2(\gamma+1)}\left\|\nabla u_{k}(t)\right\|^{2(\gamma+1)} .
\end{aligned}
$$


By using assumptions, Lemma 3.2 and the estimates (3.6), (3.10) we arrive at

$$
\begin{aligned}
& \int_{0}^{t}\left\|u_{k s}\right\|^{2} d s+\int_{0}^{t} \int_{\Omega}\left|u_{k}\right|^{q-2} u_{k s}^{2} d x d s+\frac{l}{2}\left\|\nabla u_{k}(t)\right\|^{2} \\
\leq & \text { constant independent of } k, \forall t \in[0, T] ;
\end{aligned}
$$

where $T>0$ is arbitrary large if $2<p \leq q$ and $T \in\left(0, T_{*}\right)$ if

$$
q<p<2+\frac{2 q}{n} \text {. }
$$

\section{Passing to the limit}

Let $T>0$ be an arbitrarily number if $2<p \leq q$; or $T \in\left(0, T_{*}\right)$ if

$$
q<p<2+\frac{2 q}{n} .
$$

From priori estimate (I) - (II) (see (3.10) and (3.12)), there exists function $u$ and a subsequences of $\left\{u_{k}\right\}_{k=1}^{\infty}$ that we still denote by $\left\{u_{k}\right\}_{k=1}^{\infty}$ such that, as $k \rightarrow \infty$,

$$
\begin{array}{rllll}
u_{k} & \rightarrow u & \text { weakly star in } & L^{\infty}\left(0, T ; W_{0}^{1,2(\gamma+1)}(\Omega)\right), \\
u_{k t} & \rightarrow u_{t} \quad \text { weakly in } & L^{2}\left(0, T ; L^{2}(\Omega)\right), \\
\left(\left|u_{k}\right|^{q-2}\right)_{t} & \rightarrow \chi & \text { weakly in } & L^{2}\left(0, T ; L^{2}(\Omega)\right) .
\end{array}
$$

Since the sequence $\left\{u_{k}\right\}$ is bounded in

$$
W=\left\{v \in L^{\infty}\left(0, T ; W_{0}^{1,2(\gamma+1)}(\Omega)\right): v^{\prime} \in L^{2}\left(0, T ; L^{2}(\Omega)\right)\right\}
$$

uniformly in $k$, it follows from Aubin-Lions compactness theorem that

$$
u_{k} \rightarrow u \text { strongly in } C\left(0, T ; L^{r}(\Omega)\right), \forall r \in\left[1, q^{*}\right) .
$$

Therefore, we have $u_{k}$ strongly converges to $u$ in $L^{q}(\Omega \times(0, T))$ and so, by Krasnoselskii's theorem for the Nemytskii operator we have

$$
\left|u_{k}\right|^{q / 2-2} u_{k} \longrightarrow|u|^{q / 2-2} u \text { strongly in } L^{2 q /(q-2)}(\Omega \times(0, T)) .
$$

Similarly, we also have $\left|u_{k}\right|^{q / 2}$ strongly converges to $|u|^{q / 2}$ in $L^{2}(\Omega \times(0, T))$. Therefore,

$$
\left(\left|u_{k}\right|^{q / 2}\right)_{t} \longrightarrow\left(|u|^{q / 2}\right)_{t} \text { weakly* in } D^{\prime}(\Omega \times(0, T))
$$

where $D^{\prime}(\Omega \times(0, T))$ is space of distributions on $\Omega \times(0, T)$.

By (3.15) and (3.17) we deduce that

$$
\chi=\left(|u|^{q / 2}\right)_{t} \text { and }\left(\left|u_{k}\right|^{q / 2}\right)_{t} \longrightarrow\left(|u|^{q / 2}\right)_{t} \text { weakly in } L^{2}(\Omega \times(0, T)) .
$$

Combining (3.16) and (3.18) we get

$$
\left|u_{k}\right|^{q-2} u_{k t}=\frac{2}{q}\left|u_{k}\right|^{q / 2-2} u_{k}\left(\left|u_{k}\right|^{q / 2}\right)_{t} \longrightarrow|u|^{q-2} u_{t} \quad \text { weakly in } L^{2}(\Omega \times(0, T)) .
$$

On the other hand, by the continuity of function $f$, it is obvious that

$$
f\left(u_{k}\right) \longrightarrow f(u) \text { for all }(x, t) \in(\Omega \times(0, T))
$$

Conversely, we have

$$
\int_{0}^{T} \int_{\Omega}\left|f\left(u_{k}\right)\right|^{\frac{p}{p-1}} d x d s \leq \int_{0}^{T} \int_{\Omega}\left(C_{1}+C_{2}\left|u_{k}\right|^{p-1}\right)^{p /(p-1)} d x d s \leq C_{10},
$$

for all $k$. By Lions's lemma (see [4]), it follows from (3.20) and (3.21) that

$$
f\left(u_{k}\right) \longrightarrow f(u) \quad \text { weakly in } L^{\frac{p}{p-1}}(\Omega \times(0, T)) .
$$


By (3.13)-(3.15), (3.18), (3.19) and (3.22), passing to the limit as $k \longrightarrow \infty$ in (3.3) and (3.4), we obtain that $u(x, 0)=u_{0}$,

$$
\begin{aligned}
& \int_{\Omega}\left(1+|u(t)|^{q-2}\right) u^{\prime}(t) v d x+\int_{\Omega} M\left(\|\nabla u(t)\|^{2}\right) \nabla u(t) \nabla v d x \\
& -\int_{0}^{t} g(t-s) \int_{\Omega} \nabla u(s) \nabla v d x d s \\
= & \int_{\Omega} f(u(t)) v d x
\end{aligned}
$$

for all $v \in W_{0}^{1,2(\gamma+1)}(\Omega)$ and for any $t \in[0, T]$.

This completes the proof.

\section{Blow up of solutions}

In this section, we deal with the blow up results of the solution for the problem (1.1). In order to obtain the result of blow up of solutions, we assume that following assumption:

$$
p \in\left(q, 2+\frac{2 q}{n}\right) .
$$

We denote by $C_{\rho}$ the best embedding constant for $W_{0}^{1,2(\gamma+1)}(\Omega) \hookrightarrow L^{\rho}(\Omega)$ and $C_{\rho, 1}=\frac{C_{\rho}}{\sqrt{l}}$. We put

$$
\alpha_{1}=C_{p, 1}^{-p /(p-2)} b^{-1 /(p-2)}, \quad E_{1}=\left(\frac{1}{2}-\frac{1}{p}\right) \alpha_{1}^{2}, \quad E_{2}=\left(\frac{1}{p_{1}}-\frac{1}{p}\right) \alpha_{1}^{2} .
$$

The following lemma will play an essential role in the proof of our main result in this section. It is similar to a lemma used firstly by Vitillaro ([13]). Also, the following lemma's proof can be easily established by the Lemma 3.6 in [11]. Therefore, we omit it.

Lemma 4.1. Suppose that assumptions (A1), (A2) and (A5) hold. Moreover, assume that $E(0)<E_{1}$ and $u$ is a weak solution of problem (1.1)

(i) If $\left\|\nabla u_{0}\right\|^{2}<\alpha_{1}$ then there exists $\widetilde{\alpha}_{1} \in\left[0, \alpha_{1}\right]$ such that

$$
\left(\left(1-\int_{0}^{t} g(s) d s\right)\|\nabla u(t)\|^{2}+(g \diamond \nabla u)(t)+\frac{1}{\gamma+1}\|\nabla u(t)\|^{2(\gamma+1)}\right)^{\frac{1}{2}}<\widetilde{\alpha}_{1}, \forall t \in[0, T) .
$$

(ii) If $\left\|\nabla u_{0}\right\|^{2}>\alpha_{1}$ then there exists a constant $\widehat{\alpha}_{1}>\alpha_{1}$ such that

$$
\left(\left(1-\int_{0}^{t} g(s) d s\right)\|\nabla u(t)\|^{2}+(g \diamond \nabla u)(t)+\frac{1}{\gamma+1}\|\nabla u(t)\|^{2(\gamma+1)}\right)^{\frac{1}{2}} \geq \widehat{\alpha}_{1},
$$

and

for all $t \in[0, T)$.

$$
\|u\|_{p} \geq C_{p, 1} \widehat{\alpha}_{1}
$$

Theorem 4.2. Suppose that (A1), (A2), (A4), (A5) and (A6) hold. Let $u_{0} \in W_{0}^{1,2(\gamma+1)}(\Omega)$ with

$$
0 \leq E(0)<E_{2}, \quad\left\|\nabla u_{0}\right\|>\alpha_{1} .
$$

Then the solution $u$ of the problem (1.1) blows up in finite time.

Proof. For this purpose, we set

$$
H(t)=E_{2}-E(t) .
$$

By the definition of $H(t)$ and since $E^{\prime}(t) \leq 0$, we get

$$
H^{\prime}(t)=-E^{\prime}(t) \geq 0 \text {. }
$$


Conversely, by using (2.3) and Lemma 4.1 follows that

$$
\begin{aligned}
0< & H(0) \leq H(t)=E_{2}-\frac{1}{2}\left[\left(1-\int_{0}^{t} g(s) d s\right)\|\nabla u(t)\|^{2}+(g \diamond \nabla u)(t)\right] \\
& -\frac{1}{2(\gamma+1)}\left(\|\nabla u\|^{2(\gamma+1)}\right)+\int_{\Omega} F(u) d x \\
\leq & E_{2}-\frac{1}{2} \widehat{\alpha}_{1}^{2}+\int_{\Omega} F(u) d x \\
\leq & E_{1}-\frac{1}{2} \alpha_{1}^{2}+\int_{\Omega} F(u) d x \\
= & -\frac{1}{p} \alpha_{1}^{2}+\int_{\Omega} F(u) d x .
\end{aligned}
$$

Therefore, combining ( $A 5)$ and (4.5), we have

$$
0<H(0) \leq H(t) \leq \int_{\Omega} F(u) d x \leq \frac{b}{p}\|u(t)\|_{p}^{p} .
$$

Let's define the functional

$$
\Psi(t)=H^{1-\sigma}(t)+\frac{\varepsilon}{2} \int_{\Omega}|u|^{2} d x
$$

where $\varepsilon$ small to be chosen later and

$$
0<\sigma \leq 1-\frac{q}{p}
$$

By taking a derivative of (4.7) with respect to $t$ and using Eq.(1.1), we have

$$
\begin{aligned}
\Psi^{\prime}(t)= & (1-\sigma) H^{-\sigma}(t) H^{\prime}(t)+\varepsilon \int_{\Omega} u_{t} u d x \\
= & (1-\sigma) H^{-\sigma}(t) H^{\prime}(t)-\varepsilon \int_{\Omega}|u|^{q-2} u u_{t} d x-\varepsilon\|\nabla u\|^{2}-\varepsilon\|\nabla u\|^{2(\gamma+1)} \\
& +\varepsilon \int_{0}^{t} g(t-s) \int_{\Omega} \nabla u(s) \nabla u(t) d x d s+\varepsilon \int_{\Omega} u f(u) d x .
\end{aligned}
$$

By the assumption $(A 4)$, we get

$$
\begin{aligned}
\Psi^{\prime}(t)= & (1-\sigma) H^{-\sigma}(t) H^{\prime}(t)-\varepsilon \int_{\Omega}|u|^{q-2} u u_{t} d x-\varepsilon\|\nabla u\|^{2}-\varepsilon\|\nabla u\|^{2(\gamma+1)} \\
& +\varepsilon(p-m) \int_{\Omega} F(u) d x+\varepsilon \widehat{b}\|u(t)\|_{p}^{p}+\varepsilon\left(\int_{0}^{t} g(s) d s\right)\|\nabla u\|^{2} \\
& -\varepsilon \int_{0}^{t} g(t-s) \int_{\Omega}|\nabla u(s)-\nabla u(t)||\nabla u(t)| d x d s .
\end{aligned}
$$

Furthermore, we use Young's inequality to estimate last term of right-hand of (4.9), we obtain

$$
\begin{aligned}
\Psi^{\prime}(t) \geq & (1-\sigma) H^{-\sigma}(t) H^{\prime}(t)-\varepsilon \int_{\Omega}|u|^{q-2} u u_{t} d x-\varepsilon\|\nabla u\|^{2(\gamma+1)} \\
& +\varepsilon(p-m) \int_{\Omega} F(u) d x+\varepsilon \widehat{b}\|u(t)\|_{p}^{p}-\varepsilon \delta(g \diamond \nabla u)(t) \\
& -\varepsilon\left[1-\left(1-\frac{1}{4 \delta}\right) \int_{0}^{t} g(s) d s\right]\|\nabla u\|^{2}
\end{aligned}
$$

where $\delta>0$. Then, using definition $H^{\prime}(t)$ and Young's inequality to estimate the second terms in (4.10), we get 


$$
\begin{aligned}
\int_{\Omega}|u|^{q-2} u u_{t} d x & \leq \delta_{1} \int_{\Omega}|u|^{q} d x+\frac{1}{\delta_{1}} \int_{\Omega}|u|^{q-2} u_{t}^{2} d x \\
& \leq \delta_{1}\|u(t)\|_{q}^{q}+\frac{1}{\delta_{1}} H^{\prime}(t),
\end{aligned}
$$

for all $\delta_{1}>0$. Moreover, by using (4.6) and definition of $H(t)$, we get

$$
\begin{aligned}
\|u(t)\|_{p}^{p} & \geq \frac{p}{b} \int_{\Omega} F(u) d x \\
& =\frac{p}{b}\left\{H(t)+\frac{1}{2}\left[(g \diamond \nabla u)(t)+\frac{1}{\gamma+1}\|\nabla u\|^{2(\gamma+1)}\right]\right. \\
& \left.\left.=+\frac{1}{2}\left(1-\int_{0}^{t} g(s) d s\right)\right)\|\nabla u(t)\|^{2}-E_{2}\right\}
\end{aligned}
$$

On the other hand,

$$
\begin{aligned}
& \varepsilon \widehat{b}\|u(t)\|_{p}^{p}= \varepsilon \widehat{b}\left[\frac{p-p_{1}}{p}\|u(t)\|_{p}^{p}+\frac{p_{1}}{p}\|u(t)\|_{p}^{p}\right] \\
& \geq \varepsilon \widehat{b}\left\{\frac{p-p_{1}}{p}\|u(t)\|_{p}^{p}-\frac{p_{1}}{p} \frac{p}{b} E_{2}+\frac{p_{1}}{p}\left[\frac{p}{b} H(t)+\frac{p}{2 b}(g \diamond \nabla u)(t)\right.\right. \\
&\left.\left.+\frac{p}{2 b}\left(1-\int_{0}^{t} g(s) d s\right)\|\nabla u(t)\|^{2}+\frac{p}{2 b(\gamma+1)}\|\nabla u\|^{2(\gamma+1)}\right]\right\} .
\end{aligned}
$$

and from (4.3), we have

$$
\frac{p-p_{1}}{p}\|u(t)\|_{p}^{p}-\frac{p_{1}}{p} \frac{p}{b} E_{2} \geq\left[\frac{p-p_{1}}{p}-p_{1} \frac{1}{b} E_{2}\left(C_{p, 1} \widehat{\alpha}_{1}\right)^{-p}\right]\|u(t)\|_{p}^{p} .
$$

Combining (4.14) and (4.15), we have

$$
\begin{aligned}
\varepsilon \widehat{b}\|u(t)\|_{p}^{p} \geq & \varepsilon \widehat{b}\left\{K_{0}\|u(t)\|_{p}^{p}+\frac{p_{1}}{p}\left[\frac{p}{b} H(t)+\frac{p}{2 b}(g \diamond \nabla u)(t)\right.\right. \\
& \left.\left.+\frac{p}{2 b}\left(1-\int_{0}^{t} g(s) d s\right)\|\nabla u(t)\|^{2}+\frac{p}{2 b(\gamma+1)}\|\nabla u\|^{2(\gamma+1)}\right]\right\}
\end{aligned}
$$

since $\frac{1}{b} E_{2}\left(C_{p, 1} \widehat{\alpha}_{1}\right)^{-p}<\frac{1}{b} E_{2}\left(C_{p, 1} \alpha_{1}\right)^{-p}=\frac{E_{2}}{\alpha_{1}^{2}}=\frac{p-p_{1}}{p_{1} p}, K_{0}=\frac{p-p_{1}}{p}-p_{1} \frac{1}{b} E_{2}\left(C_{p, 1} \widehat{\alpha}_{1}\right)^{-p}>0$.

Now combining (4.10), (4.11), (4.16) and let $\delta_{1}=K_{1}^{-1} H^{\sigma}(t)$, we get

$$
\begin{aligned}
\Psi^{\prime}(t) \geq & \left(1-\sigma-K_{1} \varepsilon\right) H^{-\sigma}(t) H^{\prime}(t)-\varepsilon K_{1}^{-1} H^{\sigma}(t)\|u(t)\|_{q}^{q} \\
& +\varepsilon\left(\lambda_{m} \frac{p_{1}}{2(\gamma+1)}-1\right)\left(\|\nabla u\|^{2(\gamma+1)}\right) \\
& +\varepsilon\left(p-m+\lambda_{m} p_{1}\right) H(t)+\varepsilon \widehat{b} K_{0}\|u(t)\|_{p}^{p}+\varepsilon\left(\lambda_{m} \frac{p_{1}}{2}-\delta\right)(g \diamond \nabla u)(t) \\
& +\varepsilon\left\{\lambda_{m} \frac{p_{1}}{2}\left(1-\int_{0}^{t} g(s) d s\right)-\left[1-\left(1-\frac{1}{4 \delta}\right) \int_{0}^{t} g(s) d s\right]\right\}\|\nabla u(t)\|^{2}\left({ }^{2}\right.
\end{aligned}
$$

where $\lambda_{m}=\frac{\widehat{b}(m)}{b}$ and $K_{1}>0$.

Thanks to (4.6) and embedding $L^{p}(\Omega) \hookrightarrow L^{q}(\Omega), 1 \leq q<p$, we obtain

$$
H^{\sigma}(t)\|u(t)\|_{q}^{q} \leq K_{2}\|u(t)\|_{p}^{\sigma p+q},
$$

where $K_{2}>0$ is a constant. Since $q<p$ and $0<\sigma<1-q / p$, now applying the following algebraic inequality

$$
x^{l} \leq(x+1) \leq\left(1+\frac{1}{z}\right)(x+z), \quad \forall x \geq 0,0<l<1, z>0
$$


especially, taking $x=\|u(t)\|_{p}^{p}, l=(p \sigma+q) / p, z=H(0)$, we get

$$
\begin{aligned}
\|u(t)\|_{p}^{p \sigma+q} & \leq\left(1+\frac{1}{H(0)}\right)\left(\|u(t)\|_{p}^{p}+H(0)\right) \\
& \leq\left(1+\frac{1}{H(0)}\right)\left(\|u(t)\|_{p}^{p}+H(t)\right) .
\end{aligned}
$$

Inserting (4.18) and (4.19) into (4.17), we conclude that

$$
\begin{aligned}
\Psi^{\prime}(t) \geq & \left(1-\sigma-K_{1} \varepsilon\right) H^{-\sigma}(t) H^{\prime}(t)+\varepsilon\left[p-m+\lambda_{m} p_{1}-K_{1}^{-1} K_{2}\left(1+\frac{1}{H(0)}\right)\right] H(t) \\
& +\varepsilon\left(\lambda_{m} \frac{p_{1}}{2(\gamma+1)}-1\right)\left(\|\nabla u\|^{2(\gamma+1)}\right)+\varepsilon\left(\lambda_{m} \frac{p_{1}}{2}-\delta\right)(g \diamond \nabla u)(t) \\
+ & \left.\varepsilon \widehat{b} K_{0}-K_{1}^{-1} K_{2}\left(1+\frac{1}{H(0)}\right)\right]\|u(t)\|_{p}^{p} \\
+ & \varepsilon\left\{\lambda_{m} \frac{p_{1}}{2}\left(1-\int_{0}^{t} g(s) d s\right)-\left[1-\left(1-\frac{1}{4 \delta}\right) \int_{0}^{t} g(s) d s\right]\right\}\|\nabla u(t)\|^{2} .
\end{aligned}
$$

At this point, we point out that the estimate (4.20) holds for all $m \in\left(0, p_{1}\right], K_{1}$ is large number and for all $\delta, \varepsilon>0$. By using the assumption $(A 6)$, we can choose $m$ such that

$$
\int_{0}^{t} g(s) d s<\frac{\lambda_{m} p_{1}-2}{\lambda_{m} p_{1}-2+\lambda_{m}^{-1} p_{1}^{-1}} .
$$

Then, we pick

$$
\lambda_{m} \frac{p_{1}}{2}>\max \{\delta, \gamma+1\}
$$

such that

$$
\lambda_{m}-\frac{2}{p_{1}} \frac{1-\left(1-\frac{1}{4 \delta}\right) \int_{0}^{\infty} g(s) d s}{\left.1-\int_{0}^{\infty} g(s) d s\right)}>0 .
$$

Then, we pick $K_{1}$ such that

$$
K_{1}>\max \left\{\frac{K_{2}\left(1+\frac{1}{H(0)}\right)}{p-m+\lambda_{m} p_{1}}, \frac{K_{2}\left(1+\frac{1}{H(0)}\right)}{\widehat{b} K_{0}}\right\},
$$

and then pick $\varepsilon \in\left(0, \frac{1-\sigma}{K_{1}}\right)$.

So (4.20) become

for

$$
\Psi^{\prime}(t) \geq C\left(\|u(t)\|_{p}^{p}+H(t)\right)
$$

$$
C=\varepsilon \min \left\{p-m+\lambda_{m} p_{1}-K_{1}^{-1} K_{2}\left(1+\frac{1}{H(0)}\right), \widehat{b} K_{0}-K_{1}^{-1} K_{2}\left(1+\frac{1}{H(0)}\right)\right\} .
$$

Consequently, we arrive at

$$
\Psi(t) \geq \Psi(0)>0, \forall t \geq 0 .
$$

Now, we estimate $\Psi(t)^{\frac{1}{1-\sigma}}$

$$
\begin{aligned}
\Psi^{\frac{1}{1-\sigma}}(t) & =\left(H^{1-\sigma}(t)+\frac{\varepsilon}{2}\|u(t)\|_{2}^{2}\right)^{\frac{1}{1-\sigma}} \\
& \leq C\left(H(t)+\|u(t)\|_{p}^{\frac{2}{1-\sigma}}\right) .
\end{aligned}
$$

We again apply above algebraic inequality, we get

$$
\|u(t)\|_{p}^{\frac{2}{11-\sigma}} \leq\left(1+\frac{1}{H(0)}\right)\left(\|u(t)\|_{p}^{p}+H(t)\right) .
$$


By combining of (4.21), (4.22) and (4.23) we arrive at

$$
\Psi^{\prime}(t) \geq \xi \Psi^{\frac{1}{1-\sigma}}(t),
$$

where $\xi$ is a positive constant.

A simple integration of $(4.24)$ over $(0, t)$ yields $\Psi^{\frac{\sigma}{1-\sigma}}(t) \geq \frac{1}{\Psi^{-\frac{\sigma}{1-\sigma}}(0)-\frac{\xi \sigma t}{1-\sigma}}$, which implies that the solution blows up in a finite time $T^{*}$, with

$$
T^{*} \leq \frac{1-\sigma}{\xi \sigma \Psi^{\frac{\sigma}{1-\sigma}}(0)}
$$

\section{Exponential growth of solutions}

In this section, we estimate that the energy will grow up as an exponential function as time as goes to infinity.

Theorem 5.1. Suppose that $f(u)=b|u|^{p-2} u$, (A2), (A6) hold. Let $u_{0} \in W_{0}^{1,2(\gamma+1)}(\Omega)$ with

$$
E(0)<E_{2}, \quad\left\|\nabla u_{0}\right\|>\alpha_{1} .
$$

Then there exist positive constant $C$ and $\varrho$ such that

$$
\|u(t)\|_{p} \geq C \exp (\varrho t)
$$

for all $t \in[0, T)$.

Proof. Let

$$
L(t)=H(t)+\frac{\varepsilon}{2} \int_{\Omega} u^{2}(x, t) d x,
$$

where $H(t)=E_{2}-E(t)$ and $\varepsilon$ small to be chosen later. By taking the time derivative of (5.1) and taking into account $E^{\prime}(t)$, we obtain

$$
\begin{aligned}
L^{\prime}(t) & =H^{\prime}(t)+\varepsilon \int_{\Omega} u u_{t} d x \\
& =-E^{\prime}(t)+\varepsilon \int_{\Omega} u u_{t} d x \\
& =\frac{1}{2} g(t)\|\nabla u\|^{2}-\frac{1}{2}\left(g^{\prime} \diamond \nabla u\right)(t)+\int_{\Omega} u_{t}^{2} d x+\int_{\Omega}|u|^{q-2} u_{t}^{2} d x+\varepsilon \int_{\Omega} u u_{t} d x .(5.2)
\end{aligned}
$$

By using Eq.(1.1), we get

$$
\begin{aligned}
\int_{\Omega} u u_{t} d x= & \int_{\Omega} u f(u) d x-\int_{\Omega}|u|^{q-2} u u_{t} d x-\|\nabla u(t)\|^{2(\gamma+1)} \\
& -\|\nabla u(t)\|^{2}+\int_{0}^{t} g(t-s) \int_{\Omega} \nabla u(s) \nabla u(t) d x d s \\
\geq & b\|u\|_{p}^{p}-\int_{\Omega}|u|^{q-2} u u_{t} d x-\|\nabla u(t)\|^{2(\gamma+1)} \\
& -\|\nabla u(t)\|^{2}+\int_{0}^{t} g(t-s)\|\nabla u(t)\|^{2} d s \\
& -\int_{0}^{t} g(t-s) \int_{\Omega}|\nabla u(s)-\nabla u(t)||\nabla u(t)| d x d s .
\end{aligned}
$$


In order to estimate $\int_{0}^{t} g(t-s) \int_{\Omega}|\nabla u(s)-\nabla u(t)||\nabla u(t)| d x d s$ term, using the Schwarz inequality, we obtain

$$
\begin{aligned}
\int_{\Omega} u u_{t} d x \geq & b\|u(t)\|_{p}^{p}-\left(1-\int_{0}^{t} g(t-s) d s\right)\|\nabla u(t)\|^{2}-\|\nabla u(t)\|^{2(\gamma+1)} \\
& -\int_{\Omega}|u|^{q-2} u u_{t} d x-\int_{0}^{t} g(t-s)\|\nabla u(t)\|_{2}\|\nabla u(s)-\nabla u(t)\|_{2} d s .
\end{aligned}
$$

Then, using Young's inequality to the last term of (5.4), we deduce that

$$
\begin{aligned}
\int_{\Omega} u u_{t} d x \geq & b\|u(t)\|_{p}^{p}-\left[1-\left(1-\frac{1}{4 \delta}\right) \int_{0}^{t} g(t-s) d s\right]\|\nabla u(t)\|^{2}-\|\nabla u(t)\|^{2(\gamma+1)} \\
& -\delta(g \diamond \nabla u)(t)-\int_{\Omega}|u|^{q-2} u u_{t} d x
\end{aligned}
$$

for all $\delta>0$. Insert (5.5) into (5.2), we arrive at

$$
\begin{aligned}
L^{\prime}(t) \geq & \int_{\Omega} u_{t}^{2} d x+\int_{\Omega}|u|^{q-2} u_{t}^{2} d x \\
& +\varepsilon\left[b\|u(t)\|_{p}^{p}-\left[1-\left(1-\frac{1}{4 \delta}\right) \int_{0}^{t} g(t-s) d s\right]\|\nabla u(t)\|^{2}\right. \\
& \left.-\|\nabla u(t)\|^{2(\gamma+1)}-\delta(g \diamond \nabla u)(t)-\int_{\Omega}|u|^{q-2} u u_{t} d x\right] .
\end{aligned}
$$

In order to estimate $\int_{\Omega}|u|^{q-2} u u_{t} d x$ term, applying Young's inequality and the embedding $L^{p}(\Omega) \hookrightarrow L^{q}(\Omega)$, we get

$$
\begin{aligned}
\int_{\Omega}|u|^{q-2} u u_{t} d x & \leq \alpha \int_{\Omega}|u(t)|^{q} d x+\frac{1}{\alpha} \int_{\Omega}|u|^{q-2} u_{t}^{2} d x \\
& \leq \alpha C\left(\|u(t)\|_{p}^{p}\right)^{q / p}+\frac{1}{\alpha} \int_{\Omega}|u|^{q-2} u_{t}^{2} d x,
\end{aligned}
$$

for positive embedding constant $C$. Now, again apply the above algebraic inequality and we have used the fact that $0<q / p<1$, we obtain

$$
\left(\|u(t)\|_{p}^{p}\right)^{q / p} \leq\left(1+\frac{1}{H(0)}\right)\left(\|u(t)\|_{p}^{p}+H(0)\right) .
$$

Therefore, by (5.7) and (5.8), we get

$$
\int_{\Omega}|u|^{q-2} u u_{t} d x \leq \alpha C\left(1+\frac{1}{H(0)}\right)\left(1+\frac{b}{p}\right)\|u(t)\|_{p}^{p}+\frac{1}{\alpha} \int_{\Omega}|u|^{q-2} u_{t}^{2} d x .
$$

Substituting (5.9) into (5.6), we have

$$
\begin{aligned}
L^{\prime}(t) \geq & \int_{\Omega} u_{t}^{2} d x+\left(1-\frac{\varepsilon}{\alpha}\right) \int_{\Omega}|u|^{q-2} u_{t}^{2} d x \\
& +\varepsilon\left(b-\alpha c^{\prime}\right)\|u(t)\|_{p}^{p}-\varepsilon \delta(g \diamond \nabla u)(t) \\
& -\varepsilon\left[1-\left(1-\frac{1}{4 \delta}\right) \int_{0}^{t} g(t-s) d s\right]\|\nabla u\|^{2}-\varepsilon\|\nabla u(t)\|^{2(\gamma+1)}
\end{aligned}
$$

where

By using the relation

$$
c^{\prime}=C\left(1+\frac{1}{H(0)}\right)\left(1+\frac{b}{p}\right)
$$

$$
\|u(t)\|_{p}^{p}=\frac{p}{b}\left[\begin{array}{c}
H(t)+\frac{1}{2}(g \diamond \nabla u)(t)+\frac{1}{2(\gamma+1)}\|\nabla u\|^{2(\gamma+1)} \\
+\frac{1}{2}\left(1-\int_{0}^{t} g(s) d s\right)\|\nabla u(t)\|^{2}-E_{2}
\end{array}\right],
$$


we obtain

$$
\begin{aligned}
& \varepsilon\left(b-\alpha c^{\prime}\right)\|u(t)\|_{p}^{p} \\
= & \varepsilon\left(b-\alpha c^{\prime}\right)\left(\frac{p-p_{1}}{p}\|u(t)\|_{p}^{p}+\frac{p_{1}}{p}\|u(t)\|_{p}^{p}\right) \\
= & \varepsilon\left(b-\alpha c^{\prime}\right)\left\{\frac{p-p_{1}}{p}\|u(t)\|_{p}^{p}-\frac{p_{1}}{p} \cdot \frac{p}{b} E_{2}+\frac{p_{1}}{p}\left[\frac{p}{b} H(t)+\frac{p}{2 b}(g \diamond \nabla u)(t)\right.\right. \\
& \left.\left.+\frac{p}{2 b}\left(1-\int_{0}^{t} g(s) d s\right)\|\nabla u(t)\|^{2}+\frac{p}{2 b(\gamma+1)}\|\nabla u\|^{2(\gamma+1)}\right]\right\} .
\end{aligned}
$$

Further, it follows from (4.3) that

$$
\frac{p-p_{1}}{p}\|u(t)\|_{p}^{p}-\frac{p_{1}}{p} \cdot \frac{p}{b} E_{2} \geq\left[\frac{p-p_{1}}{p}-p_{1} \frac{1}{b} E_{2}\left(C_{p, 1} \widehat{\alpha}_{1}\right)^{-p}\right]\|u(t)\|_{p}^{p} \geq 0,
$$

since

$$
\frac{1}{b} E_{2}\left(C_{p, 1} \widehat{\alpha}_{1}\right)^{-p}<\frac{1}{b} E_{2}\left(C_{p, 1} \alpha_{1}\right)^{-p}=\frac{E_{2}}{\alpha_{1}^{2}}=\frac{p-p_{1}}{p_{1} p} .
$$

From (5.11) and (5.12), we deduce that

$$
\begin{aligned}
\varepsilon\left(b-\alpha c^{\prime}\right)\|u(t)\|_{p}^{p} \geq & \varepsilon\left(1-\frac{\alpha c^{\prime}}{b}\right) \cdot \frac{p_{1}}{p}\left[p H(t)+\frac{p}{2}(g \diamond \nabla u)(t)\right. \\
& \left.+\frac{p}{2}\left(1-\int_{0}^{t} g(s) d s\right)\|\nabla u(t)\|^{2}+\frac{p}{2(\gamma+1)}\|\nabla u\|^{2(\gamma+1)}\right] .
\end{aligned}
$$

Combining (5.10) and (5.13), we obtain

$$
\begin{aligned}
L^{\prime}(t) \geq & \int_{\Omega} u_{t}^{2} d x+\left(1-\frac{\varepsilon}{\alpha}\right) \int_{\Omega}|u|^{q-2} u_{t}^{2} d x+\varepsilon p_{1}\left(1-\frac{\alpha c^{\prime}}{b}\right) H(t) \\
& +\varepsilon\left\{\frac{p_{1}}{2}\left(1-\frac{\alpha c^{\prime}}{b}\right)\left(1-\int_{0}^{t} g(s) d s\right)-\left[1-\left(1-\frac{1}{4 \delta}\right) \int_{0}^{t} g(t-s) d s\right]\right\}\|\nabla u(t)\|^{2} \\
& +\varepsilon\left[\frac{p_{1}}{2}\left(1-\frac{\alpha c^{\prime}}{b}\right)-\delta\right](g \diamond \nabla u)(t) \\
& +\varepsilon\left[\frac{p_{1}}{2(\gamma+1)}\left(1-\frac{\alpha c^{\prime}}{b}\right)-1\right]\|\nabla u(t)\|^{2(\gamma+1)}
\end{aligned}
$$

By using the assumption (A6), we can choose $\delta<p_{1} / 2$ such that

$$
1-\frac{2}{p_{1}} \cdot \frac{1-\left(1-\frac{1}{4 \delta}\right) \int_{0}^{\infty} g(t-s) d s}{1-\int_{0}^{\infty} g(s) d s}>0 .
$$

Then, we continue choosing $\alpha$ and $\varepsilon$, respectively, such that

$$
0<\alpha<\frac{b}{c^{\prime}} \min \left\{\frac{p_{1}}{2}-\delta, \frac{p_{1}}{2(\gamma+1)}-1,1-\frac{2}{p_{1}} \cdot \frac{1-\left(1-\frac{1}{4 \delta}\right) \int_{0}^{\infty} g(s) d s}{1-\int_{0}^{\infty} g(s) d s}\right\},
$$

and $\varepsilon \in(0, \alpha)$. Hence, we deduce from (5.14) that

$$
L^{\prime}(t) \geq C_{1}\left(H(t)+\|\nabla u(t)\|^{2}\right)
$$

with $C_{1}$ is a positive constant define by

$$
\begin{aligned}
C_{1}= & \varepsilon \min \left\{p_{1}\left(1-\frac{\alpha c^{\prime}}{b}\right) ; \frac{p_{1}}{2}\left(1-\frac{\alpha c^{\prime}}{b}\right)-\delta ; \frac{p_{1}}{2(\gamma+1)}\left(1-\frac{\alpha c^{\prime}}{b}\right)-1 ;\right. \\
& \left.\frac{p_{1}}{2}\left(1-\frac{\alpha c^{\prime}}{b}\right)\left(1-\int_{0}^{t} g(s) d s\right)-\left[1-\left(1-\frac{1}{4 \delta}\right) \int_{0}^{t} g(t-s) d s\right]\right\} .
\end{aligned}
$$


Moreover, by definition of $L(t)$ and Poincare's inequality, we get

$$
\begin{aligned}
L(t) & =H(t)+\frac{\varepsilon}{2} \int_{\Omega} u^{2} d x \\
& \leq H(t)+\frac{\varepsilon C_{\alpha}^{2}}{2}\|\nabla u(t)\|^{2} \\
& \leq C_{2}\left(H(t)+\|\nabla u(t)\|^{2}\right)
\end{aligned}
$$

where $C_{\alpha}$ is the Poincare constant and $C_{2}=1+\frac{\varepsilon C_{\alpha}^{2}}{2}$. Hence, by (5.15) and (5.16), we arrive at

$$
L^{\prime}(t) \geq r L(t), \quad \forall t \in[0, T) .
$$

where $r=C_{1} / C_{2}$. Integration of (5.17) between 0 and $t$ gives us the desired results.

\section{Decay of solution}

In this part, we prove the general decay of solutions of the problem (1.1). Along this part we consider both cases of $p$, that is

$$
2<p \leq q, \text { or } \quad q<p<2+\frac{2 q}{n} .
$$

Lemma 6.1 ([8]). Let $E: R^{+} \rightarrow R^{+}$be a noncreasing function; $\Theta: R^{+} \rightarrow R^{+}$be a strictly increasing function of class $C^{1}$ such that

$$
\Theta(0)=0 \text { and } \lim _{t \rightarrow+\infty} \Theta(t)=+\infty \text {. }
$$

Assume that there exist $\eta \geq 0$ and $\lambda>0$ such that

$$
\forall S \geq 0, \quad \int_{S}^{+\infty} E^{1+\eta}(t) \Theta^{\prime}(t) \leq \frac{1}{\lambda} E^{\eta}(0) E(S) .
$$

Then $E$ has the following decay properties:

$$
\begin{aligned}
& \text { If } \eta=0 \text { then } E(t) \leq E(0) \exp (1-\lambda \Theta(t)), \forall t \geq 0, \\
& \text { If } \eta>0 \text { then } E(t) \leq E(0) \exp \left(\frac{1+\eta}{1+\lambda \eta \Theta(t)}\right)^{1 / \eta}, \forall t \geq 0 .
\end{aligned}
$$

Theorem 6.2. Suppose that (A1), (A2), (A5),

$$
u f(u)-p F(u) \leq \sum_{i=1}^{M} M_{i}|u|^{p_{i}}, \forall u \in \mathbb{R},
$$

and $\forall t \geq 0$, let $\xi: R^{+} \rightarrow R^{+}$is a nonincreasing function

$$
g^{\prime}(t) \leq-\xi(t) g(t), \quad \int_{0}^{+\infty} \xi(t) d t=+\infty,
$$

where $M_{i} \geq 0$ are constants, $p_{i} \in[2, p)(i=1,2, \ldots, M)$ hold.

Assume further that the initial data $u_{0} \in W_{0}^{1,2(\gamma+1)}(\Omega)$ satisfies conditions $\left\|\nabla u_{0}\right\|_{2}<\alpha_{1}$, $0 \leq E(0)<E_{1}$, and

$$
C_{p, 1}^{p}\left(\frac{2 p}{p-2} E(0)\right)^{(p-2) / 2} b+\frac{p}{p-2} \sum_{i=1}^{M} C_{p_{i}, 1}^{p_{i}}\left(\frac{2 p}{p-2} E(0)\right)^{\left(p_{i}-2\right) / 2} M_{i}<1 .
$$

Then, solution $u$ of problem (1.1) satisfies the following energy decay estimate for some $\lambda>0$,

$$
E(t) \leq E(0) \exp \left(1-\lambda \int_{0}^{t} \xi(\tau) d \tau\right), \forall t \geq 0
$$


Proof. Firstly, we show that, for $t \geq 0$,

$$
\begin{aligned}
\ell^{1 / 2}\|\nabla u(t)\|_{2} & \leq\left[\left(1-\int_{0}^{t} g(s) d s\right)\|\nabla u(t)\|^{2}+(g \diamond \nabla u)(t)+\frac{1}{\gamma+1}\|\nabla u(t)\|^{2(\gamma+1)}\right]^{1 / 2} \\
& <\alpha_{1}=b^{-1 /(p-2)} C_{p, 1}^{-p /(p-2)} .
\end{aligned}
$$

Herefrom, we obtain

$$
\begin{aligned}
\mathcal{F}(t): & =\left(1-\int_{0}^{t} g(s) d s\right)\|\nabla u(t)\|^{2}+(g \diamond \nabla u)(t) \\
& +\frac{1}{\gamma+1}\|\nabla u(t)\|^{2(\gamma+1)}-p \int_{\Omega} F(u) d x \\
\geq & \ell\|\nabla u(t)\|^{2}-b\|u(t)\|_{p}^{p} \\
\geq & \ell\|\nabla u(t)\|^{2}-b C_{p, 1}^{p} \ell^{p / 2}\|\nabla u(t)\|^{p} \\
\geq & \ell\|\nabla u(t)\|^{2}\left(1-b C_{p, 1}^{p} \ell^{(p-2) / 2}\|\nabla u(t)\|^{p-2}\right)>0 .
\end{aligned}
$$

Conversely, using Eq.(2.3), we get

$$
E(t)=\frac{p-2}{2 p}\left[\left(1-\int_{0}^{t} g(s) d s\right)\|\nabla u(t)\|^{2}+(g \diamond \nabla u)(t)+\frac{1}{\gamma+1}\|\nabla u(t)\|^{2(\gamma+1)}\right]+\frac{1}{p} \mathcal{F}(t) .
$$

Furthermore, from (6.2) and (A5), we easily see that

$$
\ell\|\nabla u(t)\|^{2} \leq\left(1-\int_{0}^{t} g(s) d s\right)\|\nabla u(t)\|^{2} \leq \frac{2 p}{p-2} E(t) \leq \frac{2 p}{p-2} E(0) .
$$

By using embedding theorem, we get

$$
\|u(t)\|_{p}^{p} \leq C_{p}^{p}\|\nabla u(t)\|^{p} \leq \frac{2 p \vartheta_{p}}{p-2} E(t), \forall t \in[0, T),
$$

where

$$
\vartheta_{p}=C_{p, 1}^{p}\left(\frac{2 p}{p-2} E(0)\right)^{(p-2) / 2} .
$$

In the same way, we obtain

$$
\|u(t)\|_{p_{i}}^{p_{i}} \leq \frac{2 p \vartheta_{p_{i}}}{p-2} E(t), \forall t \in[0, T),
$$

with

$$
\vartheta_{p_{i}}=C_{p_{i}, 1}^{p_{i}}\left(\frac{2 p}{p-2} E(0)\right)^{\left(p_{i}-2\right) / 2}, \quad \forall i \quad(i=1,2, \ldots, M) .
$$

Let's consider the function

$$
\Theta(t)=\int_{0}^{t} \xi(s) d s, t \geq 0,
$$

which gives, as $\Theta$ is non-decreasing function of class $C^{1}$ on $\mathbb{R}^{+}$and

$$
\Theta(t) \rightarrow+\infty \text { as } t \rightarrow+\infty
$$

Multiplying Eq.(1.1) by $\xi(t) u(x, t)$ and then integrating them over $\Omega \times[S, T]$, we get

$$
\begin{aligned}
& \int_{S}^{T} \xi(t)\left\{\int_{\Omega}\left(1+|u(t)|^{q-2}\right) u u_{t} d x+\left(1-\int_{0}^{t} g(s) d s\right)\|\nabla u\|^{2}+\|\nabla u\|^{2(\gamma+1)}\right. \\
& \left.-\int_{0}^{t} \int_{\Omega} g(t-s)(\nabla u(s)-\nabla u(t)) \nabla u(t) d x d s-\int_{\Omega} f(u) u d x\right\} d t=0,
\end{aligned}
$$

where $0 \leq S<T<+\infty$. 
Taking into account the definition of $E(t)$ and (6.6), we arrive at

$$
\begin{aligned}
2 \int_{S}^{T} E(t) & \xi(t) d t=\int_{S}^{T} \xi(t)\left\{-\int_{\Omega}\left(1+|u(t)|^{q-2}\right) u u_{t} d x\right. \\
& +\int_{0}^{t} \int_{\Omega} g(t-s)(\nabla u(s)-\nabla u(t)) \nabla u(t) d x d s \\
& \left.+\int_{\Omega}[f(u) u-2 F(u)] d x-\left(1-\frac{1}{\gamma+1}\right)\|\nabla u\|^{2(\gamma+1)}+(g \diamond \nabla u)(t)\right\} d t \\
= & \int_{S}^{T} \xi(t)\left\{I_{1}+I_{2}+I_{3}-\left(1-\frac{1}{\gamma+1}\right)\|\nabla u\|^{2(\gamma+1)}+(g \diamond \nabla u)(t)\right\} d t,
\end{aligned}
$$

in what follows we will estimate $I_{1}+I_{2}+I_{3}$ in (6.7). By using Sobolev's embedding theorem, Young's inequality and (2.4), (6.3), we deduce that

$$
\begin{gathered}
I_{1} \leq \varepsilon \int_{\Omega}\left(u^{2}+|u|^{q}\right) d x+c(\varepsilon) \int_{\Omega}\left(u_{t}^{2}+|u|^{q-2} u_{t}^{2}\right) d x \\
\leq \varepsilon c E(t)-c(\varepsilon) E^{\prime}(t) . \\
I_{2} \leq \varepsilon\|\nabla u(t)\|^{2}+c(\varepsilon)\left(\int_{0}^{t} g(t-s)\|\nabla u(s)-\nabla u(t)\| d s\right)^{2} \\
\leq \varepsilon c E(t)+c(\varepsilon)\left(\int_{0}^{t} g(s) d s\right)\left(\int_{0}^{t} g(t-s)\|\nabla u(s)-\nabla u(t)\|^{2} d s\right) \\
\leq \varepsilon c E(t)+c(\varepsilon)(g \diamond \nabla u)(t) .
\end{gathered}
$$

For $I_{3}$ in (6.7), applying the assumptions (A5), since $u f(u)-p F(u) \leq \sum_{i=1}^{M} M_{i}|u|^{p_{i}}$ and (6.4)-(6.5), we get

$$
\begin{aligned}
I_{3} & =\int_{\Omega}[f(u) u-p F(u)] d x+(p-2) \int_{\Omega} F(u) d x \\
& \leq b \cdot \frac{p-2}{p} \int_{\Omega}|u|^{p} d x+\sum_{i=1}^{M} M_{i}\|u(t)\|_{p_{i}}^{p_{i}} \\
& \leq 2\left(\vartheta_{p} b+\frac{p}{p-2} \sum_{i=1}^{M} M_{i} \vartheta_{p_{i}}\right) E(t) .
\end{aligned}
$$

Then, combining these estimates (6.8)-(6.10), we arrive at

$$
\begin{aligned}
2 \int_{S}^{T} E(t) \xi(t) d t \leq & {\left[\varepsilon c+2\left(\vartheta_{p} b+\frac{p}{p-2} \sum_{i=1}^{M} M_{i} \vartheta_{p_{i}}\right)\right] \int_{S}^{T} E(t) \xi(t) d t } \\
& +c(\varepsilon) \int_{S}^{T} \xi(t)(g \diamond \nabla u)(t) d t-c(\varepsilon) \int_{S}^{T} E^{\prime}(t) \xi(t) d t .
\end{aligned}
$$

Moreover, since $g^{\prime}(t) \leq-\xi(t) g(t)$, we have

$$
\int_{S}^{T} \xi(t)(g \diamond \nabla u)(t) d t \leq-\int_{S}^{T}\left(g^{\prime} \diamond \nabla u\right)(t) d t \leq-c \int_{S}^{T} E^{\prime}(t) d t \leq c E(S) .
$$

It is obvious that

$$
-\int_{S}^{T} E^{\prime}(t) \xi(t) d t \leq \xi(0) \int_{S}^{T}-E^{\prime}(t) d t \leq c E(S)
$$

Since $\vartheta_{p} b+\frac{p}{p-2} \sum_{i=1}^{M} M_{i} \vartheta_{p_{i}}<1$ we can choose $\varepsilon$ to be a sufficent small number so that it follows from (6.13) that

$$
\int_{S}^{T} E(t) \xi(t) d t \leq c E(S)
$$


which implies

$$
\int_{S}^{+\infty} E(t) \xi(t) d t \leq c E(S), \quad \forall S \geq 0 .
$$

We end the proof by applying Lemma 6.1.

\section{References}

[1] L. Alfonsi and F. Weissler, Blow-up in $R^{n}$ for a parabolic equation with a damping nonlinear gradient term, Progr. Nonlinear Differential Equations Appl. 7, 1-20, 1992.

[2] J. Ball , Remarks on blow-up and nonexistence theorems for nonlinear evolution equations, Q. J. Math. Oxf. Ser. 28, 473-486, 1977.

[3] S.S. Dragomir, Some Gronwall Type Inequalities and Applications, RGMIA Monographs: Victoria Univ, 2002.

[4] Y. He, H. Gao and H. Wang, Blow-up and decay for a class of pseudo-parabolic pLaplacian equation with logarithmic nonlinearity, Comput. Math. Appl. 75, 459-469, 2018.

[5] V.K. Kalantarov and O. A. Ladyzhenskaya, The occurrence of collapse for quasilinear equations of parabolic and hyperbolic type, J. Sov. Math. 10, 53-70, 1978.

[6] O.A. Ladyzhenskaya, V.A. Solonnikov and N.N. Ural'tseva, Linear and quasi-linear equations of parabolic type, Translations of Mathematical Monographs, Vol. 28, Amer. Math. Soc., 1968.

[7] H. Levine, Some nonexistence and instability theorems for solutions of formally parabolic equations of the form $P u_{t}=-A u+\mathcal{F}(u)$, Arch. Ration. Mech. Anal. 51, 371-386, 1973.

[8] P. Martinez, A new method to obtain decay rate estimates for dissipative system, ESAIM Control OPTİM. Calc. Var. 4, 419-444, 1999.

[9] S.A. Messaoudi, Blow-up of semilinear heat equation with a visco-elastic term, Progr. Nonlinear Differential Equations Appl. 64, 351-356, 2005.

[10] N. Polat, Blow up of solution for a nonlinear reaction diffusion equation with multiple nonlinearities, Int. J. Sci. Technol. 2 (2), 123-128, 2007.

[11] L.X. Truong and N. Van Y, On a class of nonlinear heat equations with viscoelastic term, Comput. Math. Appl. 72, 216-232, 2016.

[12] L.X. Truong and N. Van Y, Exponential growth with $L^{p}$-norm of solutions for nonlinear heat equations with viscoelastic term, Appl. Math. Comput. 273, 656-663, 2016.

[13] E. Vitillaro, Global nonexistence theorems for a class of evolution equations with dissipation, Arch. Ration. Mech. Anal. 149, 155-182, 1999. 